\title{
Persistent spin currents in mesoscopic Haldane-gap spin rings
}

\author{
Florian Schütz, Marcus Kollar, and Peter Kopietz \\ Institut für Theoretische Physik, Universität Frankfurt, \\ Robert-Mayer-Strasse 8, 60054 Frankfurt, Germany
}

(Dated: August 12, 2003)

\begin{abstract}
Using a modified spin-wave approach, we show that in the presence of an inhomogeneous magnetic field or an in-plane inhomogeneous electric field a mesoscopic antiferromagnetic Heisenberg ring with integer spin (i.e., a Haldane-gap system) exhibits a persistent circulating spin current. Due to quantum fluctuations the current has a finite limit on the order of $\left(-g \mu_{\mathrm{B}}\right) c / L$ at zero temperature, provided the staggered correlation length $\xi$ exceeds the circumference $L$ of the ring, in close analogy to ballistic charge currents in mesoscopic normal-metal rings. Here $c$ is the spin-wave velocity, $g$ is the gyromagnetic ratio, and $\mu_{\mathrm{B}}$ is the Bohr magneton. For $\xi \ll L$ the current is exponentially suppressed.
\end{abstract}

PACS numbers: 75.10.Jm, 75.10.Pq, 75.30.Ds, 73.23.Ra

\section{INTRODUCTION}

In a recent paper, $\frac{1}{,}$ henceforth referred to as $\mathrm{I}$, we have shown that the mesoscopic persistent current in a normal-metal ring threaded by an Aharonov-Bohm flux has an analog in spin rings: specifically, we have calculated the persistent spin current $I_{s}$ in thermal equilibrium that circulates a ferromagnetic Heisenberg ring subject to a crown-shaped magnetic field with magnitude $|\boldsymbol{B}|$. Within linear spin-wave theory we have shown that the associated magnetization current $I_{m}=\left(g \mu_{\mathrm{B}} / \hbar\right) I_{s}$ (where $g$ is the gyromagnetic ratio and $\mu_{\mathrm{B}}$ is the Bohr magneton) is of the form ${ }^{2}$

$$
I_{m}=-\frac{g \mu_{\mathrm{B}}}{L} \sum_{k} \frac{v_{k}}{e^{\left(\epsilon_{k}+|\boldsymbol{h}|\right) / T}-1}
$$

where $L$ is the circumference of the ring, $\epsilon_{k}$ is the energy dispersion, and $v_{k}=\hbar^{-1} \partial \epsilon_{k} / \partial k$ is the velocity of the magnons, $|\boldsymbol{h}|=g \mu_{B}|\boldsymbol{B}|$, and $T$ is the temperature. In the presence of a crown-shaped inhomogeneous magnetic field the direction of the magnetization in the classical ground state covers a finite solid angle $\Omega_{m}$ on the unit sphere as we move once around the ring. Then the Bloch wave vectors are quantized as $k_{n}=\frac{2 \pi}{L}\left(n+\frac{\Omega_{m}}{2 \pi}\right)$, where in the continuum approximation the allowed values of $n$ are $n=0, \pm 1, \pm 2, \ldots$.

The magnetization current (1) vanishes for $T \rightarrow 0$. Physically, this is due to the fact that no quantum fluctuations are present in a ferromagnet, so that at $T=0$ there are no magnons to carry the spin current. It is thus tempting to speculate that the analogous current for an antiferromagnetic Heisenberg ring will be finite even at $T=0$ due to quantum fluctuations. In this work we shall show that this is indeed the case and present a quantitative calculation of the current in Haldane-gap antiferromagnets (i.e., antiferromagnetic Heisenberg rings with integer spin $S$ ) using a modified spin-wave theory: ${ }^{3,4,5}$ Our main result is that the ground state of a Haldane-gap spin ring subject to an inhomogeneous magnetic field supports a finite magnetization current, which in the limit where the staggered correlation length $\xi$ is large compared with

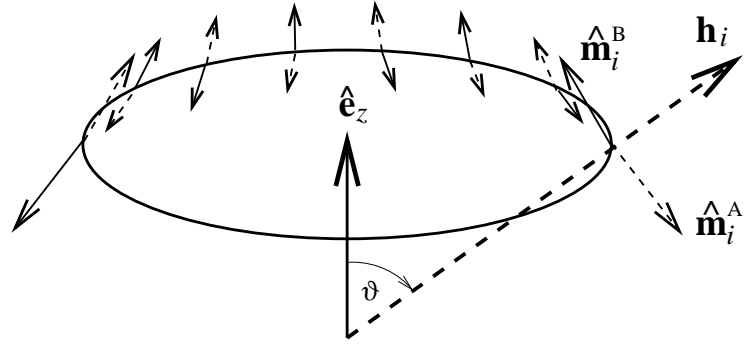

FIG. 1: Classical ground state of an antiferromagnetic Heisenberg ring in a crown-shaped magnetic field; $\boldsymbol{m}_{i}^{A}$ and $\boldsymbol{m}_{i}^{B}$ are the directions of the spins on sublattices $A$ and $B$.

$L$ has a sawtooth shape as a function of the geometric flux $\Omega$,

$$
I_{m}=\left(-g \mu_{\mathrm{B}}\right) \frac{c}{L}\left(1-\frac{\Omega}{\pi}\right), \quad 0<\frac{\Omega}{2 \pi}<1,
$$

where $c$ is the spin-wave velocity. Here $\Omega$ is the solid angle on the unit sphere traced out be the local Néel vector as one moves once around the ring. Such a state can be produced by an inhomogeneous magnetic field, as depicted in Fig. 1 Similar to the case of a ferromagnet discussed in I, the magnetization current is carried by magnons which are subject to mesoscopic interference due to the geometric phase associated with the inhomogeneous nature of the classical ground state. Due to quantum fluctuations, the ring is endowed with an electric dipole moment even in the ground state. The absence of true long-range order in one-dimensional Heisenberg antiferromagnets leads for integer spin $S$ to a finite spin-correlation length $\xi$ and a Haldane gap of the order of $\hbar c / \xi$ between the groundstate energy and the lowest triplet excitation. These features are correctly captured by a modified spin-wave theory ${ }^{3.5}$ which we use here to calculate the spin current in Haldane-gap systems. Note that this approach is only appropriate for integer $S$, where the low-energy excitations can be viewed as renormalized spin waves. In contrast, for half integer $S$ the spectrum is gapless and spin correlations decay algebraically: ${ }^{6}$ The elemen- 
tary excitations are then spinons, so that the modified spin-wave theory does not correctly reproduce the lowenergy physics. In this case the effective low-energy theory is a Tomonaga-Luttinger model. Recently Meier and Loss $\underline{7}$ used such a model to discuss the spin current in $S=1 / 2$ antiferromagnetic spin chains for a two-terminal geometry.

While Eq. (1) is the bosonic analog of the persistent charge current ${ }^{8.9}$ in a normal one-dimensional metal ring threaded by an Aharonov-Bohm flux $\phi$, Eq. (2) is formally identical with the persistent charge current in a ballistic metal ring at zero temperature. Recall that for spinless fermions at constant chemical potential $\mu$ the charge current in the ballistic regime can be written as 10

$$
I_{c}=\frac{-e}{L} \sum_{k} \frac{v_{k}}{e^{\left(\epsilon_{k}-\mu\right) / T}+1} .
$$

Here $\epsilon_{k}$ is the energy dispersion and $v_{k}$ is the corresponding velocity of an electron in the state with wave number $k$. Due to the Aharonov-Bohm flux the wave vectors are quantized as $k_{n}=\frac{2 \pi}{L}\left(n-\phi / \phi_{0}\right)$, where $\phi_{0}=h c / e$ is the flux quantum. For free fermions at zero temperature Eq. (3) reduces to a sawtooth function. For an even number of electrons at $T=0$ one obtains

$$
I_{c}=(-e) \frac{v_{F}}{L}\left(1-\frac{2 \phi}{\phi_{0}}\right), \quad 0<\frac{\phi}{\phi_{0}}<1,
$$

where $v_{F}$ is the Fermi velocity. With the replacements $e \rightarrow g \mu_{\mathrm{B}}, v_{F} \rightarrow c$, and $\phi / \phi_{0} \rightarrow \Omega / 2 \pi$ the zero-temperature charge current (44) is formally identical with the zero-temperature magnetization current (2) in a Haldane-gap spin ring. Finite temperature, disorder, and phase-breaking scattering all have a similar effect on the persistent charge current in one dimension ${ }^{10}$ For a weak perturbation, they smooth the discontinuity around $\phi=0$ and with increasing strength higher harmonics are exponentially suppressed such that a sinusoidal shape is approached. In the limit of a very strong perturbation the current is exponentially suppressed with the relevant length or energy scale, i.e., the current becomes proportional to $\exp \left(-T / T^{*}\right), \exp \left(-L / L_{\xi}\right)$, or $\exp \left(-L / L_{\phi}\right)$ under the influence of a nonzero temperature, strong disorder or strong inelastic scattering, respectively. Here $T^{*}$ is the temperature scale associated with the discrete level spacing, $L_{\xi}$ is the localization length, and $L_{\phi}$ is the phase-coherence length. Below we show that in the case of spin currents in Haldane gap systems the correlation length $\xi$ plays a similar role: for $\xi \ll L$ the magnetization current is exponentially suppressed and becomes sinusoidal.

The rest of the paper is organized as follows. In Sec. III we apply linear spin-wave theory to an antiferromagnet subject to an inhomogeneous magnetic field. After calculating the spectrum of magnon excitations, we obtain the spin current as a gauge-invariant derivative of the flux-dependent part of the free energy and discuss the result for $T=0$ in naïve spin-wave theory. To justify and generalize the spin-wave approach, we then apply a modified spin-wave theory, where the absence of long-range order in a one-dimensional antiferromagnet is taken into account. In Sec. III an antiferromagnet in an inhomogeneous electric field is considered. In Appendix A, the classical ground state is obtained explicitly for the simple geometry of a crown-shaped field, and Appendix B contains some mathematical details of the calculation of the magnetization current.

\section{SPIN-WAVE THEORY}

\section{A. Spectrum}

We start with a nearest-neighbor antiferromagnetic $(J>0)$ Heisenberg ring in an inhomogeneous magnetic field

$$
H=\sum_{i=1}^{N}\left[J \boldsymbol{S}_{i} \cdot \boldsymbol{S}_{i+1}-\boldsymbol{h}_{i} \cdot \boldsymbol{S}_{i}\right],
$$

where $\boldsymbol{S}_{i}$ are localized spin operators at sites $l_{i}$ of a onedimensional lattice with lattice spacing $a=L / N$, and $\boldsymbol{S}_{i}^{2}=S(S+1)$. Periodic boundary conditions $\boldsymbol{S}_{N+1}=\boldsymbol{S}_{1}$ are used, $\boldsymbol{h}_{i}=g \mu_{\mathrm{B}} \boldsymbol{B}_{i}$, and $N$ is assumed to be even. For a spin-wave expansion the classical ground state has to be determined by minimizing the energy in Eq. (5) with $\boldsymbol{S}_{i}$ replaced by $S \hat{\boldsymbol{m}}_{i}$, where $\hat{\boldsymbol{m}}_{i}$ is a unit vector. To consider fluctuations, the spin operators are then represented by bosonic creation and annihilation operators in the standard way,

$$
\boldsymbol{S}_{i} \cdot \boldsymbol{m}_{i}=S-b_{i}^{\dagger} b_{i}, \quad \boldsymbol{S}_{i} \cdot \boldsymbol{e}_{i}^{+}=\sqrt{2 S} b_{i}\left[1+\mathcal{O}\left(S^{-1}\right)\right],
$$

where $\boldsymbol{e}_{i}^{+}=\boldsymbol{e}_{i}^{1}+i \boldsymbol{e}_{i}^{2}$, and $\boldsymbol{e}_{i}^{1}$ and $\boldsymbol{e}_{i}^{2}$ are two unit vectors in the plane perpendicular to $\boldsymbol{m}_{i}$, such that $\left\{\boldsymbol{e}_{i}^{1}, \boldsymbol{e}_{i}^{2}, \boldsymbol{m}_{i}\right\}$ is a right-handed local basis in spin space. There is a local gauge freedom in the choice of the transverse basis vectors $\boldsymbol{e}_{i}^{1}$ and $\boldsymbol{e}_{i}^{2}$ which can be arbitrarily rotated around $\boldsymbol{m}_{i}$. With the notation of I the spin-wave Hamiltonian then reads

$$
\begin{gathered}
H_{\mathrm{sw}}=\frac{J S}{2} \sum_{i}\left\{\left[\left(1+\boldsymbol{m}_{i} \cdot \boldsymbol{m}_{i+1}\right) e^{i\left(\omega_{i \rightarrow i+1}-\omega_{i+1 \rightarrow i}\right)} b_{i}^{\dagger} b_{i+1}\right.\right. \\
\left.-\left(1-\boldsymbol{m}_{i} \cdot \boldsymbol{m}_{i+1}\right) e^{i\left(\omega_{i \rightarrow i+1}+\omega_{i+1 \rightarrow i}\right)} b_{i}^{\dagger} b_{i+1}^{\dagger}+\text { h.c. }\right] \\
\left.-2 \boldsymbol{m}_{i} \cdot \boldsymbol{m}_{i+1}\left(b_{i}^{\dagger} b_{i}+b_{i+1}^{\dagger} b_{i+1}\right)+\boldsymbol{h}_{i} \cdot \boldsymbol{m}_{i} b_{i}^{\dagger} b_{i}\right\}, \quad \text { (7) }
\end{gathered}
$$

where $\omega_{i \rightarrow j}$ is the angle of rotation around $\boldsymbol{m}_{i}$ that takes $\boldsymbol{m}_{i} \times \boldsymbol{m}_{j}$ into $\boldsymbol{e}_{i}^{1}$. The transformation to another local right-handed triad, associated with site $i$ and the bond (ij) according to $\left\{\tilde{\boldsymbol{e}}_{i}^{1}, \tilde{\boldsymbol{e}}_{i}^{2}=\boldsymbol{m}_{i} \times \boldsymbol{m}_{j}, \boldsymbol{m}_{i}\right\}$, then reads

$$
\boldsymbol{e}_{i}^{ \pm}=e^{ \pm i \omega_{i \rightarrow j}} \tilde{\boldsymbol{e}}_{i}^{ \pm}
$$

Equation (7) is manifestly invariant under the $\mathrm{U}(1)$ gauge transformation $\omega_{i \rightarrow j} \rightarrow \omega_{i \rightarrow j}+\alpha_{i}, b_{i} \rightarrow e^{i \alpha_{i}} b_{i}$. We will 
now assume a sufficiently large ring so that the direction of the magnetic field varies only slightly on the scale of the lattice spacing $a$. The classical ground state then locally resembles a Néel state and the local Néel vector $\boldsymbol{n}_{i}=(-1)^{i+1} \boldsymbol{m}_{i}$ varies smoothly as a function of position on the lattice and is oriented almost orthogonal to the local direction of the magnetic field. We thus have $\boldsymbol{m}_{i} \cdot \boldsymbol{m}_{i+1}=-1+O(1 / N)$ and the terms involving the combination $b_{i}^{\dagger} b_{i+1}$ in Eq. (7) can be neglected to leading order in $1 / N$. In this approximation a local spin deviation follows the direction of the classical ground state as it moves around the ring. It picks up a geometrical phase leading to interference of its wave function in close analogy to Aharonov-Bohm interference in charge transport. The effect of the inhomogeneous field can be incorporated via the gauge transformation

$$
\alpha_{j}=\sum_{i=1}^{j-1}(-1)^{j+i}\left(\omega_{i \rightarrow i+1}+\omega_{i+1 \rightarrow i}\right) .
$$

The new boundary conditions then become in the bosonic language

$$
b_{i+N}=e^{ \pm i \Omega} b_{i}, \quad \Omega=\alpha_{N+1}
$$

where the upper/lower sign is valid for sublattice $A / B$ (odd/even $i$ ). The resulting quadratic bosonic Hamiltonian is standard for an antiferromagnetic ring with nearest-neighbor interactions

$$
H_{\mathrm{AFM}}=\sum_{i}\left[-J S\left(b_{i} b_{i+1}+b_{i}^{\dagger} b_{i+1}^{\dagger}\right)+\left(2 J S+h_{s}\right) b_{i}^{\dagger} b_{i}\right],
$$

except for the twisted boundary condition (10). An additional staggered field $h_{s}$ in the direction of the classical ground-state vectors has been introduced as a technical tool for the discussion in Sec. IIC $H_{\mathrm{AFM}}$ is diagonalized as usual by first performing Fourier transformations with different signs on the two sublattices:

$$
a_{k}=\sqrt{\frac{2}{N}} \sum_{i \in A} e^{-i k l_{i}} b_{i}, \quad b_{k}=\sqrt{\frac{2}{N}} \sum_{i \in B} e^{+i k l_{i}} b_{i}
$$

where the allowed wave vectors are given by

$$
k_{n}=\frac{2 \pi}{L}\left(n+\frac{\Omega}{2 \pi}\right), \quad n=0, \ldots, \frac{N}{2}-1 .
$$

The diagonal form of $H_{\mathrm{AFM}}$ is then achieved by the Bogoliubov transformation

$$
\left(\begin{array}{c}
a_{k} \\
b_{k}^{\dagger}
\end{array}\right)=\left(\begin{array}{cc}
\cosh \theta_{k} & \sinh \theta_{k} \\
\sinh \theta_{k} & \cosh \theta_{k}
\end{array}\right)\left(\begin{array}{c}
\alpha_{k} \\
\beta_{k}^{\dagger}
\end{array}\right)
$$

with

$$
\tanh \left(2 \theta_{k}\right)=\frac{\cos (k a)}{1+\tilde{h}_{s}}, \quad \tilde{h}_{s}=h_{s} / 2 J S
$$

The diagonal Hamiltonian contains constant terms due to quantum fluctuations,

$$
H_{\mathrm{AFM}}=\sum_{k} \epsilon_{k}\left(\alpha_{k}^{\dagger} \alpha_{k}+\beta_{k}^{\dagger} \beta_{k}+1\right)-N J S\left(1+\tilde{h}_{s}\right)
$$

where the quasiparticle energies are given by

$$
\epsilon_{k}=2 J S \sqrt{\Delta^{2}+\sin ^{2}(k a)}, \quad \Delta^{2}=\tilde{h}_{s}\left(\tilde{h}_{s}+2\right),
$$

and the free energy is obtained from

$$
F_{\mathrm{sw}}(\Omega)=2 T \sum_{k} \ln \left[2 \sinh \frac{\epsilon_{k}}{2 T}\right]-N J S\left(1+\tilde{h}_{s}\right) .
$$

Thus we have shown that to leading order in $1 / N$ thermodynamic quantities depend on the inhomogeneity of the field only via the single phase $\Omega$. Geometrically $\Omega$ is the anholonomy associated with the parallel transport of a vector orthogonal to the local Néel vector, 11 in analogy to the ferromagnetic case. To see this more clearly, we consider for each bond $(i j)$ two additional sets of local right-handed triads containing the Néel vector $\boldsymbol{n}_{i}$ instead of $\boldsymbol{m}_{i}$. These triads are given by $\left\{\overline{\boldsymbol{e}}_{i}^{1}=\boldsymbol{e}_{i}^{1}, \overline{\boldsymbol{e}}_{i}^{2}=\right.$ $\left.(-1)^{i+1} \boldsymbol{e}_{i}^{2}, \boldsymbol{n}_{i}\right\}$ and $\left\{\tilde{\overline{\boldsymbol{e}}}_{i}^{1}, \tilde{\overline{\boldsymbol{e}}}_{i}^{2}=\boldsymbol{n}_{i} \times \boldsymbol{n}_{j}, \boldsymbol{n}_{i}\right\}$, and are related by a rotation around $\boldsymbol{n}_{i}$. For the associated spherical vectors this reads

$$
\overline{\boldsymbol{e}}_{i}^{ \pm}=e^{ \pm i \bar{\omega}_{i \rightarrow j}} \tilde{\overline{\boldsymbol{e}}}_{i}^{ \pm}
$$

where the rotation angles $\bar{\omega}_{i \rightarrow j}$ are given by

$$
\bar{\omega}_{i \rightarrow j}=i \pi+(-1)^{i+1} \omega_{i \rightarrow j} \quad \text { for } \quad j=i \pm 1 .
$$

We can now express $\Omega$ as

$$
\Omega=\sum_{i=1}^{N}\left(\bar{\omega}_{i \rightarrow i+1}-\bar{\omega}_{i+1 \rightarrow i}\right) \quad \bmod 2 \pi
$$

which is of the form obtained in I for the ferromagnet. $\Omega$ is thus the anholonomy of a vector orthogonal to the local Néel vector that is transported around the ring by discrete rotations around $\boldsymbol{n}_{i} \times \boldsymbol{n}_{i+1}$. Alternatively, a continuous parallel transport can be used around a path of geodesics connecting the unit vectors $\boldsymbol{n}_{i}$ on the unit sphere. $\Omega$ is therefore equal to the solid angle subtended by this closed path of geodesics.

\section{B. Magnetization current}

In I we have shown that the $\mathrm{U}(1)$ gauge symmetry associated with the choice of the local transverse basis is connected with a conserved current that was identified with the longitudinal component of the spin current. For an antiferromagnet the longitudinal spin current is conveniently defined in the direction of the local Néel vector 
and can be written as a gauge-invariant derivative of the free energy

$$
I_{s}=\left\langle\boldsymbol{n}_{i} \cdot \boldsymbol{I}_{i \rightarrow i+1}\right\rangle=-(-1)^{i+1}\left\langle\frac{\partial H_{\mathrm{sw}}}{\partial \omega_{i \rightarrow i+1}}\right\rangle=-\frac{\partial F_{\mathrm{sw}}}{\partial \Omega},
$$

where $\boldsymbol{I}_{i \rightarrow i+1}=J \boldsymbol{S}_{i} \times \boldsymbol{S}_{i+1}$ is the spin current from site $i$ to $i+1$. Similar to the ferromagnetic case, the presence of a longitudinal spin current can be understood semiclassically as follows. The local spin deviation from the classical ground state is essentially perpendicular to $\boldsymbol{n}_{i}$ and varies slightly from $i$ to $i+1$. The spin deviations on neighboring sites are therefore in a plane that does not contain $\boldsymbol{n}_{i}$, so that their cross product appearing in $\boldsymbol{I}_{i \rightarrow i+1}$ has a nonvanishing component $I_{s}$ in the direction of $\boldsymbol{n}_{i}$. This spin current $I_{s}$ corresponds to a current of magnetic dipoles that are locally oriented in the direction of the Néel vector $\boldsymbol{n}_{i}$, which varies smoothly as we move along the ring. The spin current generates an electric dipole field which has the same form as discussed in I. For the magnetization current we obtain

$$
I_{m}=\frac{g \mu_{\mathrm{B}}}{\hbar} I_{s}=-\frac{2 g \mu_{\mathrm{B}}}{L} \sum_{k} c_{k}\left[n_{k}+\frac{1}{2}\right]
$$

where $c_{k}=\hbar^{-1} \partial \epsilon_{k} / \partial k$ is the velocity of a magnon with wave vector $k$ and $n_{k}=1 /\left[\exp \left(\epsilon_{k} / T\right)-1\right]$ is the Bose occupation factor. The extra factor of 2 is due to the two degenerate spin-wave modes. Equation (23) is the antiferromagnetic spin analog of Eq. (3). It clearly shows that the magnetization current is carried by antiferromagnetic magnons, which at this level of approximation are the only quasiparticles available for transport. The current has a finite limit, even for vanishing Bose occupation factors, due to quantum fluctuations. From Eq. (23) the current is clearly seen to be a periodic function of $\Omega$, so that the finite momentum sum can be further evaluated using the Poisson summation formula $\stackrel{10}{10}$ Some details of the calculation are given in Appendix [B] For a vanishing staggered field in the zero-temperature limit we obtain the simple result announced in the Introduction,

$$
I_{m}=I_{m}^{0}\left(1-\frac{\Omega}{\pi}\right), \quad 0<\frac{\Omega}{2 \pi}<1 .
$$

Here $I_{m}^{0}=-g \mu_{\mathrm{B}} c / L$ is the magnetization current carried by a single magnon with the spin-wave velocity $c=c_{k \rightarrow 0^{+}}$at the center of the Brillouin zone. The sawtooth shape (see the solid line in Fig. 22) of the current in Eq. (24) is reminiscent of Eq. (41) for charge transport. Indeed, for $T=0$ Eq. (23) is formally equivalent to Eq. (31) for charge transport when the Fermi edge is replaced by the lower edge of the magnon band.

\section{Modified spin-wave theory}

The usual spin-wave theory employed so far is inconsistent when zero modes appear. Although the spin current

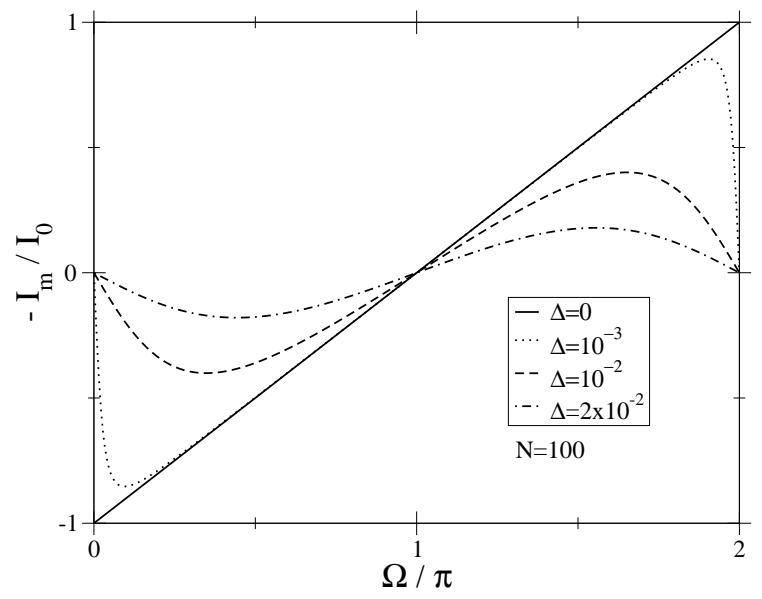

FIG. 2: Magnetization current in a ring with 100 spins for different values of the energy-gap parameter $\Delta$. The plots are produced by numerically evaluating Eq. (23). For $\Delta=10^{-3}$ the curve is indistinguishable from the approximate expression in Eq. (28), and Eq. (29) provides a good approximation for $\Delta=2 \times 10^{-2}$.

remains finite, the sublattice magnetization diverges in the limit $\Omega \rightarrow 0$. This failure is related to the absence of long-range order in one-dimensional systems. It can be resolved by a modified spin-wave theory which was first used by Takahashi for a one-dimensional ferromagnet ${ }^{3}$ and then extended to various spin systems without longrange order, including antiferromagnets $\underline{\underline{4}}$ The constraint that is introduced in these theories was recently shown to follow naturally from a calculation at constant order parameter $\stackrel{\underline{5}}{\underline{5}}$ In the present context, we introduce the additional constraint

$$
\sum_{i}\left\langle\boldsymbol{S}_{i} \cdot \boldsymbol{m}_{i}\right\rangle=0
$$

which suppresses Néel order on average. This constraint is enforced via the staggered field $h_{s}$ in Eq. (11) which acts as a Lagrange multiplier. The expectation value in Eq. (25) can be evaluated from $\partial F_{\mathrm{sw}} / \partial h_{s}$, yielding the self-consistency condition

$$
\frac{2}{N} \sum_{k} \frac{\partial \epsilon_{k}}{\partial h_{s}}\left[n_{k}+\frac{1}{2}\right]=S+\frac{1}{2} .
$$

Although the self-consistently determined $h_{s}$ is itself a periodic function of the geometric flux $\Omega$, the leading order for large $N$ is a constant and can be determined by replacing the sum in Eq. (26) by an integral. For $T=0$ the solution of Eq. (26) yields the Haldane gap $2 J S \Delta$, which is inversely proportional to the staggered correlation length $\xi, \frac{6}{\underline{6}}$

$$
\Delta=4 e^{-\pi(S+1 / 2)}=\frac{a}{\sqrt{2} \xi} .
$$

The functional form of the magnetization current shows a crossover between the two qualitatively different regimes 
$\xi \gg L$ and $\xi \ll L$ (see Fig. 2). In the former case $\Delta \ll 2 \pi / N$ and at most one wave vector can be in the region $-\Delta<k<\Delta$ where the dispersion relation deviates strongly from the dispersion in the limit $\Delta=0$. When the contribution from this single wave vector is taken into account separately, we obtain

$$
\frac{I_{m}}{I_{m}^{0}}=\frac{\sin (2 \Omega / N)}{2 \sqrt{\Delta^{2}+\sin ^{2}(\Omega / N)}}-\frac{\Omega}{\pi}, \quad-\pi<\Omega<\pi .
$$

In the case $\Delta=0$ this reduces to Eq. (24), provided $N \gg 1$. Thus, the effect of a finite $\Delta$ is to remove the discontinuity at $\Omega=0,2 \pi$. On the other hand, in the limit $\xi \ll L$ many $k$ values are affected by the energy gap. An analytic result for the spin current can nevertheless be derived as described in Appendix B. We obtain the scaling form

$$
\frac{I_{m}}{I_{m}^{0}}=\sqrt{\frac{2}{\pi}}\left(\frac{L}{\sqrt{2} \xi}\right)^{1 / 2} \exp \left(-\frac{L}{\sqrt{2} \xi}\right) \sin (\Omega),
$$

implying that the sinusoidal magnetization current is exponentially suppressed in the bulk limit, $L \gg \xi$.

\section{ELECTRIC FIELD}

Moving magnetic dipoles represent an electric dipole moment $\frac{12}{12}$ and are therefore affected by electric fields. Due to this relativistic effect, which is essentially equivalent to spin-orbit coupling, the magnetic moments acquire an Aharonov-Casher phase $\stackrel{13}{13}$ For localized spin systems described by a Heisenberg Hamiltonian, the electric field can be taken into account phenomenologically by a substitution in the interaction term,

$$
\boldsymbol{S}_{i} \cdot \boldsymbol{S}_{j} \longrightarrow \boldsymbol{S}_{i} \cdot e^{\boldsymbol{\theta}_{i j} \times} \boldsymbol{S}_{j},
$$

as long as the electric field varies only weakly on the scale of the lattice spacing. Here,

$$
\boldsymbol{\theta}_{i j}=\frac{g \mu_{\mathrm{B}}}{\hbar c^{2}} \int_{l_{i}}^{l_{j}} d \boldsymbol{l} \times \boldsymbol{E}(\boldsymbol{l}),
$$

and $e^{\boldsymbol{\theta} \times}$ denotes the $\mathrm{SO}(3)$ rotation matrix acting on a vector $\boldsymbol{m}$ according to 14

$$
e^{\boldsymbol{\theta} \times \boldsymbol{m}}=\hat{\boldsymbol{\theta}}(\hat{\boldsymbol{\theta}} \cdot \boldsymbol{m})+(\hat{\boldsymbol{\theta}} \times \boldsymbol{m}) \sin \theta-\hat{\boldsymbol{\theta}} \times(\hat{\boldsymbol{\theta}} \times \boldsymbol{m}) \cos \theta,
$$

with $\boldsymbol{\theta}=\theta \hat{\boldsymbol{\theta}}$. For ferromagnetic coupling, inhomogeneous electric fields can lead to persistent magnetization currents ${ }^{15}$ and a spin analog of the Hall effect was also shown to exist in electric fields ${ }^{7}$

We now consider the antiferromagnetic ring in an electric field in the $x-y$ plane, e.g. produced by a charged line in the $z$ direction (see Fig. 3i). The rotation vectors $\boldsymbol{\theta}_{i, i+1}=\theta_{i, i+1} \boldsymbol{e}^{z}$ are then all parallel to the $z$ axis and the Hamiltonian for vanishing magnetic field becomes

$$
H=J \sum_{i}\left[\frac{1}{2}\left(e^{i \theta_{i, i+1}} S_{i}^{+} S_{i+1}^{-}+\text {h.c. }\right)+S_{i}^{z} S_{i+1}^{z}\right] .
$$

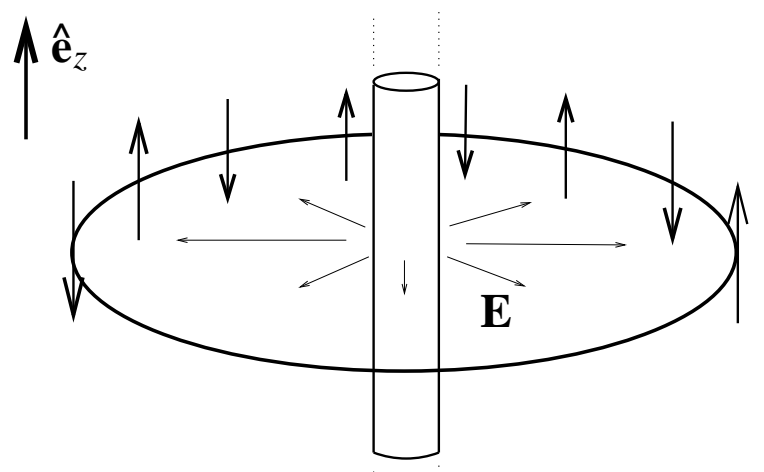

FIG. 3: Antiferromagnetic Heisenberg ring in the electric field produced by a line charge.

The classical ground state is easily shown to be a doubly degenerate Néel state with $\boldsymbol{m}_{i}= \pm \boldsymbol{e}^{z}$. The spin-wave expansion is thus straightforward. If a gauge transformation is used to eliminate the phase factors, we again obtain the standard bosonic Hamiltonian $H_{\mathrm{AFM}}$ of Eq. (11) with the boundary condition (10), where $\Omega$ is replaced by the total Aharonov-Casher phase

$$
\Omega_{\mathrm{AC}}=\sum_{i} \theta_{i, i+1}=\frac{g \mu_{\mathrm{B}}}{\hbar c^{2}} \oint d \boldsymbol{l} \cdot\left[\boldsymbol{e}^{z} \times \boldsymbol{E}(\boldsymbol{l})\right] .
$$

The spin current then only has a $z$ component which can be written as a gauge-invariant derivative of the free energy,

$$
I_{m}=-\frac{\partial F_{\mathrm{AFM}}}{\partial \Omega_{\mathrm{AC}}} .
$$

This leads again to Eq. (23) with $\Omega$ replaced by $\Omega_{\mathrm{AC}}$ and all the results derived in the previous sections are also applicable in this context.

It is also interesting to note that the situation of a radial electric field with $\theta_{i, i+1}=\frac{2 \pi}{N}$ and an additional homogeneous magnetic field tilted with respect to the $z$ axis can be formally mapped onto a crown-shaped magnetic field alone via the transformation

$$
\boldsymbol{S}_{i}^{\prime}=e^{\frac{2 \pi}{N} e^{z} \times} \boldsymbol{S}_{i} .
$$

It would therefore be interesting to further investigate the combined effect of arbitrary inhomogeneous magnetic and electric fields on the produced spin currents to find situations that could be realized more easily in the laboratory for a possible experimental detection of the effect.

\section{SUMMARY AND OUTLOOK}

In the last two decades, a lot of theoretical work focused on persistent equilibrium currents in mesoscopic normal-metal rings $\stackrel{8.9 .16}{=}$ In the $1990 \mathrm{~s}$, the experimental difficulties for the detection of the currents were 
overcome and an oscillating magnetization as a function of the magnetic flux was observed under various conditions $17 \cdot 18 \cdot 19.20 .21 .22$ In the ballistic regime, the main features of the experiment ${ }^{19}$ can be understood within a simple model of free fermions,$\frac{10}{1}$ but in the diffusive regime a generally accepted explanation of the experiments 17,18 is still lacking.

In this work, we have shown that persistent magnetization currents are present in antiferromagnetic Heisenberg rings in inhomogeneous magnetic fields as well as in a radial electric field. Quantum fluctuations lead to groundstate currents, but fluctuations in low dimensions also produce exponential damping when the circumference $L$ of the ring becomes larger than the staggered correlation length. We have obtained explicit expressions for the current at $T=0$ in the two limits $L \ll \xi$ and $L \gg \xi$ within a modified spin-wave approach valid for integer spins, i.e., for Haldane-gap systems. The determination of the current for half-integer spin rings remains an interesting open problem. Also, we have only considered clean systems in this work, i.e., we have focused on the ballistic regime. Since for persistent charge current disorder is known to be important, it would also be very interesting to consider persistent magnetization currents in the diffusive regime of disordered magnets.

In the past few years a new field of research ("spintronics") has emerged, where the spin degree of freedom is used as a medium to transport information ${ }^{23}$ For technical applications (such as quantum computation) it is important to control the quantum coherence in mesoscopic spin systems. The persistent spin current discussed in this work can be viewed as a quantitative measure for the degree of quantum coherence in the ground state of the system. Similar to the ferromagnetic case discussed in I, the magnetization current circulating a Heisenberg antiferromagnet generates an electric dipole field. Due to screening effects, the corresponding voltage drop may be rather difficult to detect experimentally. However, in view of the rapid development of the field of spintronics, it does not seem unreasonable to expect that before the end of this decade new experimental techniques will be available to detect persistent spin currents in Heisenberg rings.

We thank Florian Meier and Bernd Wolf for discussions. This work was supported by the DFG via Forschergruppe FOR 412, Project No. KO 1442/5-1.

\section{APPENDIX A: CROWN-SHAPED MAGNETIC FIELD}

Here we determine the classical ground state of an antiferromagnetic ring in the crown-shaped magnetic field depicted in Fig. 11 i.e., a field with

$$
\boldsymbol{h}_{i}=h\left[\sin (\vartheta) \cos \left(2 \pi l_{i} / L\right), \sin (\vartheta) \sin \left(2 \pi l_{i} / L\right), \cos (\vartheta)\right]
$$

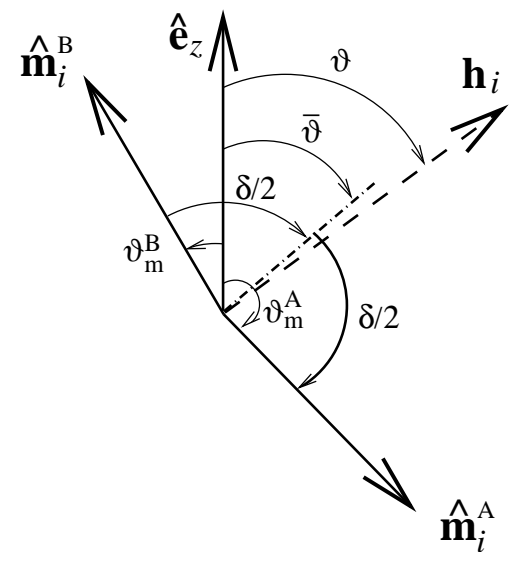

FIG. 4: Definition of angles for the description of the classical ground state of an antiferromagnetic Heisenberg ring in a crown-shaped magnetic field.

For very strong magnetic fields the classical unit vectors $\boldsymbol{m}_{i}$ will be aligned parallel to the field and the ground state will have the full rotational symmetry of the applied field. Below a critical spin-flip field $h_{c}(\vartheta)$ it will be energetically favorable to form two sublattices with different angles $\vartheta_{m}^{A / B}$ to the $z$-axis (see Fig. 4). Introducing the relative and average angles

$$
\delta=\frac{1}{2}\left(\vartheta_{m}^{A}-\vartheta_{m}^{B}\right) \quad \bar{\vartheta}=\frac{1}{2}\left(\vartheta_{m}^{A}+\vartheta_{m}^{B}\right)
$$

a minimum of the classical energy is reached for

$$
\begin{aligned}
& \sin (\vartheta-\bar{\vartheta}) \cos (\delta)=-\frac{J S}{h} \epsilon_{-} \sin (2 \bar{\vartheta}) \\
& \cos (\vartheta-\bar{\vartheta}) \sin (\delta)=+\frac{J S}{h} \epsilon_{+} \sin (2 \delta),
\end{aligned}
$$

where we have defined $\epsilon_{ \pm}=1 \pm \cos (2 \pi / N)$. For very strong magnetic fields $h>h_{c}$, we have $\delta=0$ and Eq. A3 reduces to its ferromagnetic analogue (see Eq. (14) in I). For $\delta \neq 0$ the two equations can be combined to give

$$
\sin (2(\vartheta-\bar{\vartheta}))=-\left(\frac{2 J S}{h}\right)^{2} \sin ^{2}(2 \pi / N) \sin (2 \bar{\vartheta})
$$

Thus for large rings the magnetic field $h \sim J S / N$ necessary to produce an inhomogeneous classical ground state is well below the spin-flip field $h_{c} \sim J S$. For $h \sim J S / N \ll J S$ we have $\delta \sim \pi / 2$ and the classical ground state locally resembles a Néel state as assumed in Sec. III

\section{APPENDIX B: CALCULATION OF SPIN CURRENT}

In this appendix we present some details of the calculation of the functional form of the spin current at zero 
temperature. Eq. 23) can be written in the form

$$
\frac{I_{m}}{I_{m}^{0}}=\sum_{n=0}^{N / 2-1} f\left(\frac{2 \pi}{N}\left(n+\frac{\Omega}{2 \pi}\right)\right),
$$

where $f(k)=d / d(k a)\left(\epsilon_{k} / 2 J S\right)$ is a periodic function of its argument with $f(x+\pi)=f(x)=-f(-x)$. Consequently $I_{m}$ is a periodic function of $\Omega$ and we can proceed by calculating its Fourier coefficients $C_{\nu}$ in

$$
\frac{I_{m}}{I_{m}^{0}}=\sum_{\nu=-\infty}^{\infty} C_{\nu} e^{i \nu \Omega}
$$

By appropriate substitution in the corresponding integral one eliminates the finite momentum sum and obtains

$$
C_{\nu}=-\frac{\nu N^{2} \Delta}{2 i} g_{\nu N}\left(-\Delta^{-2}\right)
$$

where $g_{2 l+1}$ is zero and $g_{2 l}$ is given by

$$
\begin{aligned}
g_{2 l}(z) & =\int_{0}^{2 \pi} \frac{d \omega}{2 \pi} \sqrt{1-z \sin ^{2} \omega} e^{-i 2 l \omega} \\
& =\left(\begin{array}{c}
\frac{1}{2} \\
l
\end{array}\right)\left(\frac{z}{4}\right)^{l}{ }_{2} F_{1}\left(l-\frac{1}{2}, l+\frac{1}{2} ; 2 l+1 ; z\right) \\
& =(-1)^{l} \sqrt{1-z} g_{2 l}\left(\frac{z}{z-1}\right) .
\end{aligned}
$$

Using an asymptotic expansion of the hypergeometric function ${ }^{24}$ for large $l$, we derive an expression for the spin current for large system sizes. In particular, the condition that the leading term in the expansion be sufficient can be shown to be equivalent to $L / \xi \gg \mathcal{O}(1)$. After some algebra we obtain Eq. (29).
1 F. Schütz, M. Kollar, and P. Kopietz, Phys. Rev. Lett. 91, 017205 (2003).

2 The extra minus sign in Eq. (1) in comparison to Eq. (15) of Ref. 1 is due to a different sign convention in the Fourier transformation used in the present work.

3 M. Takahashi, Prog. Theor. Phys. Suppl. 87, 233 (1986); Phys. Rev. Lett. 58, 168 (1987).

4 M. Takahashi, Phys. Rev. B 40, 2494 (1989); J. E. Hirsch and S. Tang, Phys. Rev. B 40, 4769 (1989).

5 M. Kollar, I. Spremo, and P. Kopietz, Phys. Rev. B 67, 104427 (2003).

${ }^{6}$ See, for example, A. Auerbach, Interacting Electrons and Quantum Magnetism (Springer, New York, 1994).

7 F. Meier and D. Loss, Phys. Rev. Lett. 90, 167204 (2003)

8 M. Büttiker, Y. Imry, and R. Landauer, Phys. Lett. 96A, 365 (1983).

9 Y. Imry, Introduction to Mesoscopic Physics (Oxford University Press, Oxford, 1997).

${ }^{10}$ H.-F. Cheung, Y. Gefen, E. K. Riedel, and W. H. Shih, Phys. Rev. B 37, 6050 (1988); H.-F. Cheung, Y. Gefen, and E. K. Riedel, IBM J. Res. Dev. 32, 359 (1988).

11 See, for example, A. Shapere and F. Wilczek, Geometric Phases in Physics, (World Scientific, Singapore, 1989).

12 J. E. Hirsch, Phys. Rev. B 60, 14787 (1999).

13 Y. Aharonov and A. Casher, Phys. Rev. Lett. 53, 319 (1984).
14 P. Chandra, P. Coleman, and A. I. Larkin, J. Phys.: Condens. Matter 2, 7933 (1990).

15 Z. Cao, X. Yu, and R. Han, Phys. Rev. B 56, 5077 (1997).

16 For a recent review, see U. Eckern and P. Schwab, J. of Low Temp. Phys. 126, 1291 (2002).

17 L. P. Lévy, G. Dolan, J. Dunsmuir, and H. Bouchiat, Phys. Rev. Lett. 64, 2074 (1990).

18 V. Chandrasekhar, R. A. Webb, M. J. Brady, M. B. Ketchen, W. J. Gallagher, and A. Kleinsasser, Phys. Rev. Lett. 67, 3578 (1991).

19 D. Mailly, C. Chapelier, and A. Benoît, Phys. Rev. Lett. 70, 2020 (1993).

20 B. Reulet, M. Ramin, H. Bouchiat, and D. Mailly, Phys. Rev. Lett. 75, 124 (1995).

21 W. Rabaud, L. Saminadayar, D. Mailly, K. Hasselbach, A. Benoît, and B. Etienne, Phys. Rev. Lett. 86, 3124 (2001).

22 E. M. Q. Jariwala, P. Mohanty, M. B. Ketchen, and R. A. Webb, Phys. Rev. Lett. 86, 1594 (2001).

23 See, for example, Semiconductor Spintronics and Quantum Computation, edited by D. D. Awschalom, D. Loss and N. Samarth (Springer Verlag, Berlin, 2002).

24 M. Abramowitz, and I. A. Stegun, Handbook of Mathematical Functions, (Dover Publications, New York, 1970); G. N. Watson, Trans. Cambr. Phil. Soc. 22, 36 (1918). 